\title{
La réalité virtuelle au service de l'apprentissage de la géométrie descriptive : retour vers Monge
}

\author{
Virtual reality \& descriptive geometry learning: Back to \\ Monge
}

Nawri Khamallah*, Renaud Pleitinx, Luca Sgambi
UCLouvain - LOCI Tournai, Rue du Glategnies, 6, 7500 Tournai

Résumé. Cet article s'intéresse aux avantages pédagogiques de la réalité virtuelle et à son application dans le cas de l'apprentissage de la géométrie descriptive. Il décrit l'implémentation et les premiers résultats d'une application en réalité virtuelle destinée à soutenir l'enseignement de la géométrie descriptive dans la Faculté d'architecture, d'ingénierie architecturale, d'urbanisme de l'UCLouvain.

Mots-clés. Géométrie descriptive, Réalité virtuelle, Architecture.

\begin{abstract}
This article focuses on the pedagogical advantages of virtual reality and its application in the case of descriptive geometry learning. It describes the implementation and first results of a virtual reality application to support the teaching of descriptive geometry in the Faculty of Architecture, Architectural Engineering and Urban Planning of UCLouvain.
\end{abstract}

Keywords. Descriptive geometry, Virtual reality, Architecture.

\section{Introduction}

Cet article s'intéresse aux avantages pédagogiques de la réalité virtuelle et à son usage dans le cas de l'apprentissage de la géométrie descriptive. Il décrit l'implémentation et les premiers résultats de l'application GéDeRéV (géométrie descriptive en réalité virtuelle), destinée à soutenir l'enseignement de la géométrie descriptive dans la Faculté d'architecture, d'ingénierie architecturale, d'urbanisme de l'UCLouvain ${ }^{1}$.

\footnotetext{
* Corresponding author: nawri.khamallah@uclouvain.be

${ }^{1}$ Le projet Géométrie descriptive et Réalité virtuelle est réalisé avec le soutien du financier du Fonds de développement pédagogique de l'UCLouvain en 2018.
} 
La première partie de cet article est consacrée à une revue des différents usages de la $\mathrm{RV}$ dans des contextes privés et professionnels et plus particulièrement dans un cadre pédagogique. La seconde partie est, quant à elle, consacrée, d'une part, à une brève présentation de la géométrie descriptive et à ses difficultés d'apprentissage et, d'autre part, à une présentation du projet Géométrie descriptive et Réalité virtuelle.

\section{Les utilisations de la RV}

Les outils de la réalité virtuelle (RV; en anglais virtual reality [VR]) permettent de plonger une personne dans un monde artificiel créé numériquement. Elle ne doit pas être confondue avec la réalité augmentée, qui superpose des éléments virtuels au monde réel. Introduites par les chercheurs de l'Université de l'Utah dans les années 1970, les premières expérimentations de spatialisation virtuelle se sont développées durant les années 1990 et 2000 dans le secteur du jeu vidéo. Aujourd'hui, les potentialités de l'immersion sont mises à profit dans les secteurs professionnels (médical, industriel, architectural) et pédagogiques.

\subsection{Utilisations ludiques et professionnelles}

\subsubsection{Usages ludiques}

Le secteur du jeu vidéo, médium adapté à des explorations narratives spatiales, est le plus propice au développement de la technologie de la RV et de sa diffusion auprès du grand public. C'est pourquoi les casques commerciaux lancés en 2016 (HTC Vive, Gear VR, Oculus Rift...) avaient pour cible le public du secteur vidéo ludique.

Aujourd'hui, le domaine des réseaux sociaux et d'internet est pris d'assaut par une nouvelle variété d'acteurs. Initié par l'expérience vidéosociale Second Life (Figure 1), le cyberespace a réémergé ces derniers mois avec la mainmise de Facebook sur l'une des principales entreprises de réalité virtuelle, Oculus, à travers laquelle elle essaie d'y introduire des éléments d'interaction sociale.

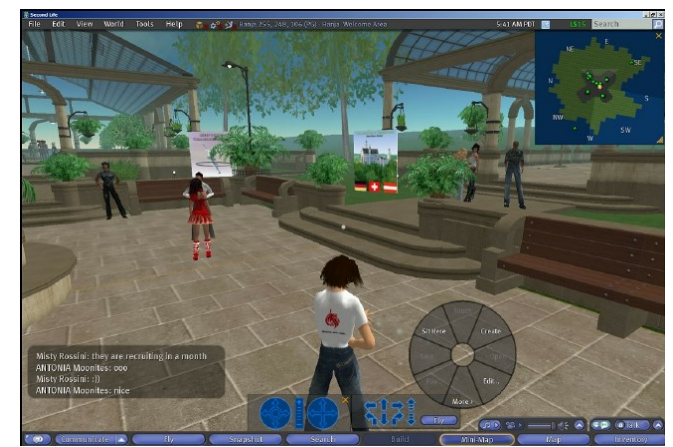

Figure 1. Capture d'écran du jeu vidéo Second Life, de Linden Lab.

Ces applications sociales rendent possible le fait d'interagir avec d'autres utilisateurs au travers d'un avatar. La mouture modernisée et contemporaine de Second Life, VR-Chat, application sortie en 2016, offre les mêmes possibilités d'interactions sociales grâce à une ergonomie et un gameplay intuitifs (McVeigh-Schultz \& Márquez, 2018). 


\subsubsection{Exploitations professionnelles}

Grâce à la simulation visuelle, la RV permet de nombreuses applications médicales. Premièrement, elle est utilisée dans le traitement du stress, l'ennui ou la dépression. La RV permet de s'évader du quotidien, surtout pour les personnes hospitalisées durant de longues périodes, mais aussi avant les opérations. Immergé dans un autre monde ou dans un jeu, le cerveau se laisse distraire des événements immédiats. Deuxièmement, la RV offre une solution pour traiter les douleurs. D'après une étude clinique menée par Cedars-Sinai, et réalisée sur 100 patients, une baisse de $24 \%$ des scores de douleurs a été observée chez ceux ayant regardé une vidéo sur un casque RV (Spiegel, Fuller \& Lopez, 2019). La RV permet de tromper le cerveau. Dans le cas de personnes amputées et souffrant du syndrome du « membre fantôme ", par exemple, on fait croire au système nerveux central, que le membre est toujours là, grâce à des exercices équipés d'un casque de RV, permettant aux patients de visualiser l'intégralité de leur corps. Troisièmement, la RV a fait ses preuves dans le traitement des phobies et des dépendances. Le traitement des phobies par la RV, en particulier, présente l'avantage d'être plus progressif que les méthodes de confrontation directes utilisées jusqu'ici (Lambrey, Jouvent, Allilaire \& Pélissolo, 2011), tout en offrant une maîtrise complète des événements et des paramètres aux thérapeutes. Après les attentats du 11 septembre 2001 à New York, la RV a été utilisée pour traiter les syndromes post-traumatiques des victimes (Difede \& Hoffman, 2002).

Outre son utilisation dans le secteur médical, la RV est actuellement utilisée dans le monde industriel. Le prototypage virtuel et les logiciels d'ingénierie virtuelle permettent aux ingénieurs et aux développeurs de produits d'accélérer le temps de leur développement, et aux entreprises de mettre leurs produits sur le marché plus rapidement et de manière plus rentable, tout en garantissant que ces nouveaux produits répondent à toutes les normes en vigueur. L'introduction de la RV dans le secteur industriel améliore, en outre, le processus de conception des produits en permettant à toutes les personnes impliquées dans le projet de les visualiser et de tester leur comportement. Les concepteurs peuvent, par exemple, monter dans voiture virtuelle pour apprécier la manière dont leurs conceptions se comporteront dans le monde physique. (Martin-Erro, Alfonso \& Espinosa, 2014)

La RV connaît un essor dans le secteur de l'architecture. Grâce aux casques de RV comme l'Oculus Rift ou le HTC Vive, les architectes peuvent désormais utiliser la RV pour accéder à des méthodes de travail et de création plus efficaces. Cette technologie permet notamment de visualiser un bâtiment et ses dimensions avec plus de précision qu'un rendu traditionnel, de percevoir les relations entre les différentes pièces et de communiquer plus facilement avec les clients. Aujourd'hui, les architectes et autres acteurs du bâtiment ont accès à de nombreux logiciels de type "plug-in », tels que Lumion, Twinmotion, ou l'Unreal Engine, qui leur permettent de visualiser l'édifice en cours de conception voire de réalisation.

\subsection{Utilisations pédagogiques de la réalité virtuelle}

Par stimulations visuelles de l'intégralité du champ oculaire, par les stimuli sonores et tactiles, la RV est un outil pédagogique efficace, adapté à de nombreuses situations d'apprentissage. L'application OssoVR, par exemple, permet l'apprentissage d'actes médicaux (Kim, Kim \& Kim, 2017) dans un environnement médicalisé interactif. De nombreuses expériences pédagogiques se sont servies des dispositifs de réalité augmentée et de RV développés par Microsoft avec successivement l'Hololens puis l'Hololens 2 (Hockett \& Ingleby, 2016). Le dispositif Hyve-3D (Figure 2), en particulier, permet un 
apprentissage et une collaboration collective. Remplaçant la simulation virtuelle individuelle générée par un écran et deux lentilles par un dispositif de vidéoprojecteurs orientés sur une surface sphérique, ce dispositif permet à toute une assemblée de pouvoir assister aux expériences virtuelles ${ }^{2}$.

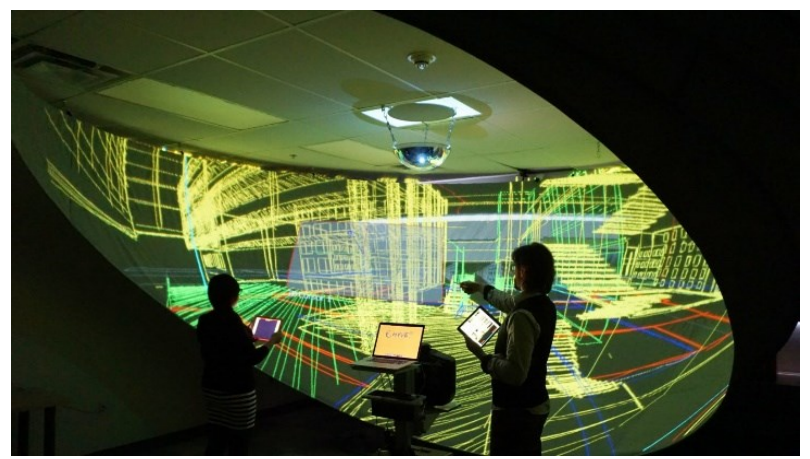

Figure 2. Hyve3D, du Laboratoire de recherche en design Hybridab.

La RV offre en particulier deux possibilités d'une grande valeur pédagogique : celle de pouvoir visiter des espaces inaccessibles (imaginaires, disparus, lointains, impénétrables) et celle de voir et de manipuler des concepts.

\subsubsection{Visiter des espaces inaccessibles}

La RV permet de visiter des espaces imaginaires. Aujourd'hui, cette technologie est particulièrement populaire dans l'exploration immersive des maquettes en trois dimensions des projets architecturaux. L'application Enscape, par exemple, permet à tout moment du processus de conception de visualiser les projets d'architecture, dans toutes les conditions de luminosité ou d'intempéries, ou d'interchanger en temps réel des éléments de projet comme la composition spatiale d'une pièce ou la matérialité d'un mur (Zeile \& Zelling, 2019).

La RV et les technologies immersives ont en outre l'avantage de permettre la visite virtuelle d'espaces lointains. Il est, par exemple, possible de visiter, sans quitter son domicile, une reconstitution de la Casa Mila de Gaudi. Il est aussi possible de poser un pied sur la lune, grâce à de nombreuses applications, plus ou moins abouties, telles qu'Apollo 11 VR.

La RV offre aussi la possibilité de visiter des espaces disparus. Aujourd'hui, la combinaison de ces technologies et des technologies d'acquisition de données permet de reconstituer de façon accessible des visites de vestiges archéologiques, comme avec le projet Tournai Map 3D qui permet de superposer deux simulations de la ville de Tournai, respectivement en 1701 et en $2016^{3}$, ou encore avec le projet de la reconstitution virtuelle

\footnotetext{
${ }^{2}$ Hyve3D est un exemple intéressant de dispositif pédagogique et collaboratif. En l'appliquant à des questions de simulation visuelle architecturale et d'interaction à travers des outils inédits, il démontre que de nouveaux champs de perception neuro-ergonomiques sont à envisager dans le champ de l'immersion architecturale.

3 "Le nuage et la ville" - Découvrez la reconstitution 3D de Tournai de 1701 à nos jours!,

UCLouvain, Tournai, 2017, vidéo https://www.youtube.com/watch?v=bh2LKbnzkrE
} 
de la Maison du Peuple de Victor Horta $^{4}$ initié par le laboratoire AllCe de la Faculté d'architecture La Cambre-Horta. Il est aussi envisageable de pouvoir visiter des espaces reconstitués, car détruits comme le pavillon allemand de Mies van der Rohe, à la suite de son démontage vis-à-vis de l'exposition internationale de 1929.

Enfin, la RV permet de visiter des espaces impénétrables. L'application développée par GE Healthcare en 2017 (Figure 3), par exemple, permet de se mouvoir dans un corps humain, de le sectionner, d'en modifier l'échelle, d'en retirer des couches, et ainsi de comprendre sa complexité anatomique.

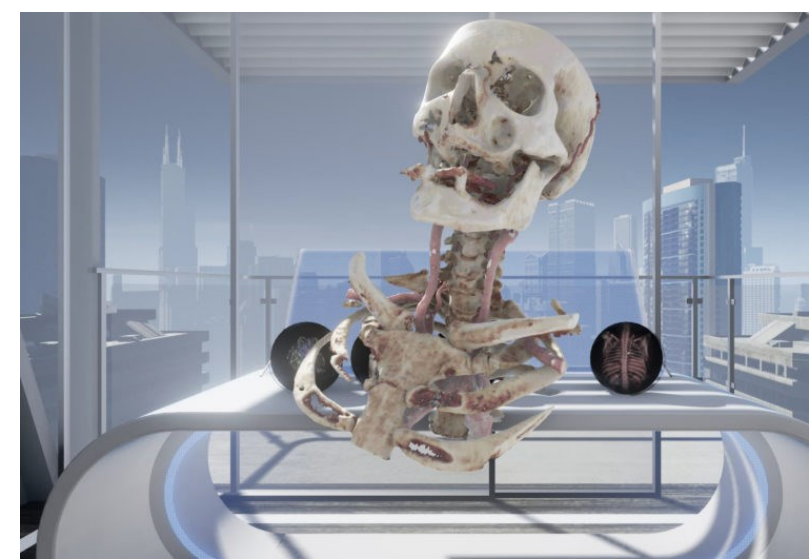

Figure 3. Capture d'écran du programme développé par General Electrics Healthcare, en 2017.

\subsubsection{Voir et manipuler des concepts}

La RV permet aussi de voir et de manipuler des concepts. La RV permet, par exemple, de représenter et manipuler des structures chimiques. Le projet Sampling molecular conformational dynamics in virtual reality, présenté dans la revue Science Advances (O’Connor, Deeks, Dawn \& Metatla, 2018) propose un environnement de travail collaboratif dans lequel plusieurs utilisateurs peuvent manipuler des molécules complexes en même temps. Les chercheurs rapportent que grâce à ce dispositif en $\mathrm{RV}$, ils peuvent étudier de nombreux problèmes d'agencement moléculaire comme les sites de liaison chimique d'une molécule pharmaceutique, le repliement des protéines, voire les combinaisons impliquées dans des réactions chimiques.

\section{Géométrie descriptive et réalité virtuelle}

Le développement de la RV, son exploitation dans le secteur architectural et ses avantages pédagogiques évoqués ci-dessus invitent à l'utilisation de cette technologie dans le cadre de l'initiation à la visualisation spatiale au début de l'enseignement de l'architecture.

Le projet de développement pédagogique Géométrie descriptive et réalité virtuelle vise à fournir aux étudiant·e·s en architecture un logiciel didactique en Réalité virtuelle (RV)

\footnotetext{
${ }^{4}$ Reconstitution virtuelle de la Maison du peuple de Victor Horta, ULB, Bruxelles, https://actus.ulb.be/fr/actus/enseignement/la-reconstitution-virtuelle-de-la-maison-du-peuple-devictor-horta
} 
permettant de réviser, en autonomie, la matière du cours de Géométrie Descriptive (GD), dans un espace d'apprentissage immersif, interactif et attractif à l'intérieur duquel les objets en $3 \mathrm{D}$ et leurs représentations en $2 \mathrm{D}$ seront simultanément visibles et manipulables.

\subsection{Géométrie descriptive}

\subsubsection{L'enseignement de la géométrie descriptive}

Systématisée par le mathématicien français Gaspard Monge, la GD est un procédé graphique dont le but est de représenter (projeter) sans ambiguité les objets (points, lignes, surfaces, volumes) d'un espace à trois dimensions sur un support à deux dimensions (Monge 1811 : viii).

Fixant les principes du dessin industriel, la GD est un prérequis pour l'apprentissage d'autres modes de représentation, tels que les perspectives obliques ou coniques, couramment utilisées par les architectes. Ayant pour effet d'accroître les compétences spatiales (Gittler \& Glück 1998), la GD est traditionnellement enseignée dans les premières années de formation des architectes. À l'UCLouvain en particulier, la GD fait l'objet de deux cours spécifiques, donnés dans le cadre du programme de Bachelier en architecture sur les sites de Bruxelles et de Tournai (LBARC1120, LBARC1121, LTARC1120, LTARC1121), qui totalisent 6 crédits et concernent chaque année $+/-250$ étudiant·e·s.

\subsubsection{Difficultés de la géométrie descriptive}

L'apprentissage de la GD, à LOCI Tournai comme à LOCI Bruxelles, se fait de façon classique, sur table, avec les outils de l'architecte utilisés avant l'apparition généralisée de l'outil informatique (papier, té, équerre, crayons, et stylos à encre de type Rotring Rapidograph). En complément, sont pratiqués lors des travaux pratiques de GD des exercices nécessitant la fabrication par les étudiant $\cdot e \cdot s$ de maquettes, supports des énoncés, rendant plus ludique la compréhension des notions enseignées. D'autres facultés d'architecture combinent enseignement analogique et expérimentation numérique comme à l'EPFL avec le logiciel propriétaire TopSolid (Cache, 2016), ou à l'ENSAM avec le logiciel libre GeoGebra. Les facultés d'architecture LOCI se démarquent elles par la volonté de ne pas utiliser l'outil informatique durant les cours de GD de première année, qui se veut exclusivement dédiée à l'acquisition de capacités de visualisation dans l'espace. Cette orientation permet aux enseignant $\cdot e \cdot s$ de construire des ponts pédagogiques. Ponts entre la GD et les cours de moyens d'expression (dessin à main levée, croquis), les cours de mathématiques, et enfin avec l'enseignement du projet d'architecture qui se fait aussi par choix pédagogique sans utilisation de l'outil informatique.

L'apprentissage de la GD présente des difficultés notables dont témoignent non seulement les résultats obtenus aux cours consacrés à cette matière ainsi que les erreurs de représentation relevées régulièrement sur les documents graphiques produits dans le cadre des ateliers d'architecture, mais aussi, et surtout, le renouvellement régulier et la diversification des supports et procédés pédagogiques que mettent en place les enseignants de cette matière pour la rendre plus accessible. Ces difficultés tiennent principalement à un manque de visualisation immédiate et de manipulation intuitive de la projection orthogonale sur laquelle repose la GD. « Une bonne maitrise des opérations projectives est nécessaire à la réussite de l'apprentissage du dessin technique, aussi bien que de la géométrie descriptive. ” (Pavel \& Ribeiro Pola 1998). Peu efficaces, les schémas couramment utilisés pour expliquer les principes de la projection orthogonale montrent en 
perspective les différents éléments de la GD (objets dans l'espace à trois dimensions, dièdre de projection et ligne de terre, plans et axes de projections, représentations en deux dimensions et lignes de rappel), mais ils n'offrent qu'un seul point de vue sur ce dispositif et ne sont habituellement pas manipulables. En outre, recourant à des représentations à deux dimensions pour expliquer un dispositif qui en possède trois, elles supposent acquises les notions et les savoir-faire que les apprenant·e's sont justement censé $\cdot e \cdot s$ assimiler et acquérir.

\subsection{Géométrie descriptive et réalité virtuelle}

Pour pallier les difficultés indiquées ci-dessus et rendre plus aisé l'apprentissage de la GD, le projet Géométrie descriptive et réalité virtuelle (GDRV) a pour objectif d'élaborer, de produire et de tester un logiciel didactique contenant un module de formation en géométrie descriptive tirant parti du caractère immersif et ludique de la RV. Si la littérature fait état de nombreuses utilisations d'outils multimédias dans le cadre de l'apprentissage de la GD (Chu, Jeng \& Chen 2015, García, Quirós, Santos, \& al. 2007, Teixeira, da Silva \& da Silva 2000), il n'existe pas, à ce jour d'outils pédagogiques immersifs destinés à l'enseignement de la GD, bien que des études récentes aient prouvé l'efficacité d'une exploitation de la RV à des fins pédagogiques (Greenwald, Kulik, Kunert, \& al. 2017, Gwee 2013, Merchant, Goetz, Cifuentes, \& al. 2014).

\subsubsection{Présentation du projet}

Le projet GDRV poursuit un objectif didactique, d'une part, et objectif technologique, d'autre part.

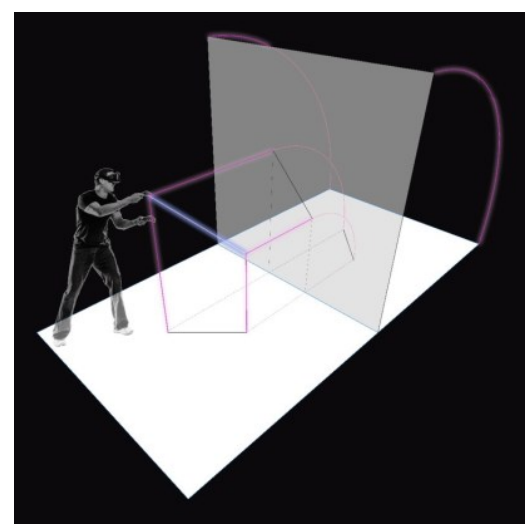

Figure 4. Dessin de synthèse de la philosophie du projet, par les auteurs.

L'objectif didactique est de fournir aux étudiant·e·s un module de formation en GD, complémentaire à un enseignement ex cathedra et visant un approfondissement, en autonomie, de la matière. A desseins, le module de formation contient des rappels théoriques et des exercices qui sont délivrés dans un espace virtuel interactif simulant l'espace de projection géométrale, propre à la GD. Immergés dans cet espace, les étudiant $\cdot e \cdot s$ peuvent, directement et simultanément, voir et manipuler, au moyen de lunettes et de manettes 3D, l'ensemble des éléments constitutifs de la GD (Figure 4). Ils peuvent ainsi éprouver corporellement les deux opérations abstraites que sont la projection et son inverse, le relèvement, et ainsi améliorer leur " vision dans l'espace ». L'élaboration du contenu didactique du module se fonde sur les syllabus de cours de GD donnés en LOCI et 
tire parti des méthodes d'enseignement éprouvées. Une attention particulière est portée au respect des conventions graphiques (figure 5).
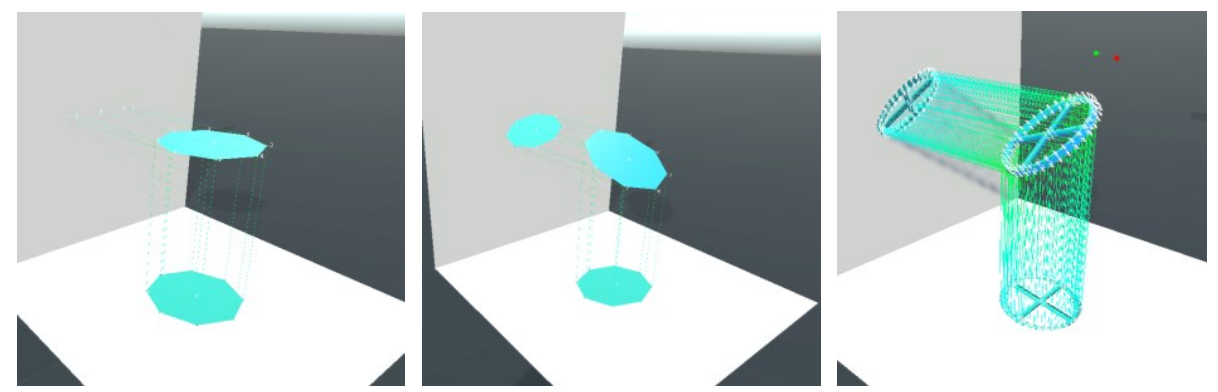

Figure 5. Captures d'écran du projet GDRV : explication visuelle de la translation d'une figure et du tracé par projection, par les auteurs.

L'objectif technologique du projet GéDeRéV est de développer d'un logiciel ergonomique d'usage intuitif et attractif exploitable ou téléchargeable en ligne, au travers d'une interface dépouillée. En termes de produit, il s'agit de développer une application tirant parti d'outils existants qui ont fait leurs preuves sur le marché de la RV, à savoir : Unity, un des 2 principaux moteurs temps réel et dispose d'un programme de licence gratuite pour l'enseignement et VRTK une librairie open source permettant de prototyper rapidement des applications de RV avec Unity, moteur de jeu vidéo trois dimensions en temps réel. Le choix d'un moteur en constante évolution se justifie par une volonté de disposer d'une application traversant les systèmes informatiques et les prochaines générations de matériel en RV et augmentée. Le projet est donc compatible avec une multitude de configurations de type PC, et la quasi-totalité des casques de RV disponibles sur le marché (de l'Oculus Rift en passant par les casques Windows Mixed Reality en passant par les derniers HTC Vive Cosmos). Ce logiciel est réalisé en collaboration avec MYRON Software, entreprise spécialisée dans le développement de jeux vidéo en RV.

L'application GéDeRéV n'a pas pour vocation de se substituer à l'épure (le dessin) et d'éviter l'effort d'abstraction qu'elle implique, mais bien de rendre plus aisé l'apprentissage des notions de base de la GD. Celle-ci est nécessaire à la visualisation par les étudiant $\cdot \mathrm{e} \cdot \mathrm{s}$ architectes des éléments abstraits à court terme à travers les propriétés fondamentales de la géométrie spatiale. La GD permet aussi de réaliser manuellement des croquis d'objets dans l'espace, puis enfin de pratiquer avec davantage de compétence les logiciels informatiques 3D contemporains (Jaques \& Calame, 2013). L'utilisation de la réalité virtuelle devra donc permettre de diminuer la pénibilité de l'effort de visualisation des épures et du trièdre, mais aussi de créer des liens pédagogiques plus évidents avec l'utilisation de l'outil informatique qui continue ensuite la formation en architecture. 


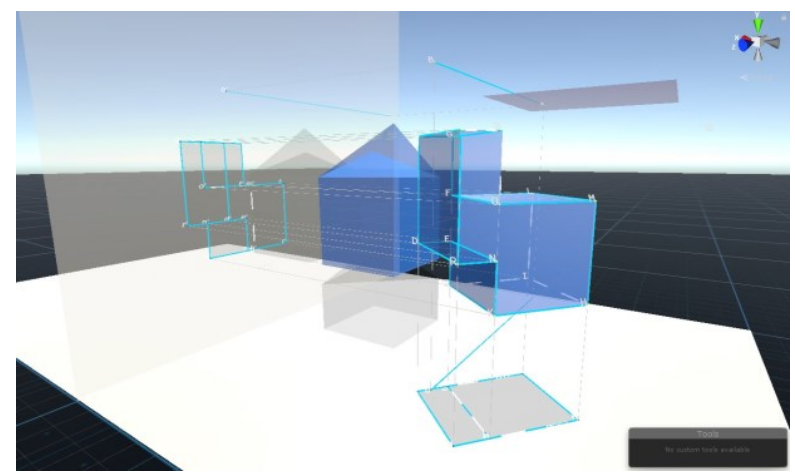

Figure 6. Capture d'écran du projet GDRV, transposition d'une épure simple, par les auteurs.

\subsubsection{Première évaluation}

En cours de développement, l'application a fait l'objet d'une première évaluation auprès de 16 étudiant·e's volontaires inscrits en première année de BAC. L'échantillon se composait de 6 filles et de 10 garçons, qui ont testé l'application durant quinze minutes, avec un dispositif HTC-Vive. L'exercice consistait à faire observer aux étudiant $\cdot e \cdot s$ une épure de géométrie descriptive simple, puis de visualiser dans l'application GéDeRéV (figure 7) cette même épure. Après avoir essayé l'application, les étudiant·e·s ont répondu à un questionnaire qui visait à rendre compte de la connaissance et de la maîtrise initiale qu'avaient les étudiants de la RV et de la géométrie descriptive, à évaluer la maniabilité et l'apport pédagogique de l'application et à juger de la valeur ajoutée de la RV en matière de GD.

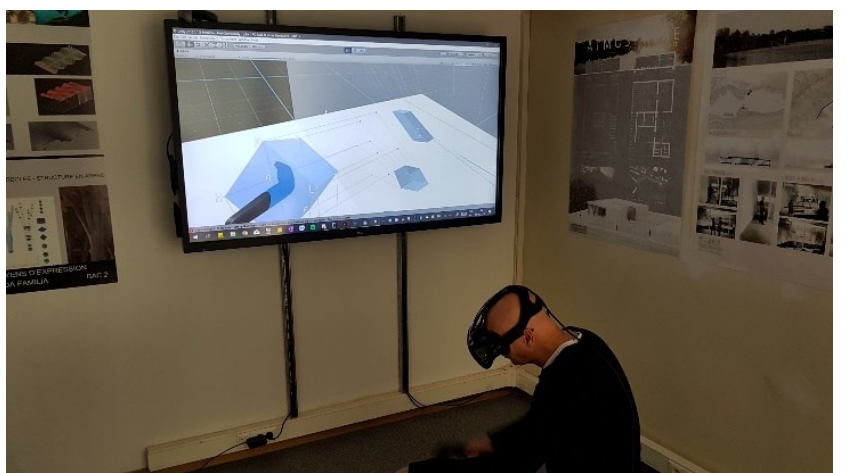

Figure 7. Photo d'une phase de test du projet GéDeRéV sur environnement de développement Unity, par les auteurs. 
Tableau 1. Retours étudiants de la faculté LOCI, UCLouvain, sur un panel de 16 étudiants durant une expérimentation de 15 minutes, 1 désignant faible et 10 désignant forte.

\begin{tabular}{|c|c|c|c|c|c|c|c|c|c|c|}
\hline & 1 & 2 & 3 & 4 & 5 & 6 & 7 & 8 & 9 & 10 \\
\hline Connaissance de la RV & $\mathbf{2}$ & $\mathbf{4}$ & $\mathbf{3}$ & $\mathbf{3}$ & 1 & 0 & 0 & 0 & 0 & 3 \\
\hline Maîtrise de la GD & 2 & 1 & $\mathbf{3}$ & 2 & 0 & 0 & $\mathbf{3}$ & 1 & 0 & $\mathbf{4}$ \\
\hline Maniabilité de l'application & 0 & 0 & 0 & 0 & 0 & 0 & $\mathbf{2}$ & $\mathbf{2}$ & $\mathbf{2}$ & $\mathbf{1 0}$ \\
\hline $\begin{array}{c}\text { Intérêt de l'expérience par } \\
\text { rapport à la matière initiale }\end{array}$ & 0 & 0 & 0 & 0 & 0 & 0 & $\mathbf{3}$ & $\mathbf{1}$ & $\mathbf{2}$ & $\mathbf{1 0}$ \\
\hline $\begin{array}{c}\text { Pertinence et apport de la } \\
\text { RV }\end{array}$ & 0 & 0 & 0 & 0 & 0 & 0 & $\mathbf{3}$ & $\mathbf{1}$ & $\mathbf{2}$ & $\mathbf{1 0}$ \\
\hline
\end{tabular}

L'enquête (tableau 1) a permis d'enregistrer une réaction globalement positive des utilisateurs. Les étudiant·e's ont démontré une bonne compréhension des principes de la projection dans l'espace du géométral en rapport avec l'exercice initial présenté par l'épure. Ils ont en outre témoigné de l'avantage pédagogique que présentent la visualisation et la manipulation simultanée des objets (3D) dans le dièdre et de leur représentation (2D) en plan.

Lorsque l'application sera opérationnelle, ses effets sur l'apprentissage de la GD feront l'objet d'un test. Les étudiant·e's de premier cycle de bachelier seront divisés en deux groupes, dont un seulement sera invité à utiliser l'application GéDeRéV, et l'autre servira de groupe témoin, sans utilisation de l'application développée. Les deux groupes seront interrogés sur les notions enseignées en début et en fin de période d'apprentissage, et leurs résultats aux deux épreuves seront comparés.

Ce test permettra une évaluation plus fine, et pourra donner des indications pour donner suite à l'acquisition des parties théoriques en plus de la manipulation libre, de dégager des conclusions sur l'efficacité et les spécificités du dispositif dans l'apprentissage des représentations mentales dans l'espace, en complémentent des enseignements classiques de GD.

\section{Conclusion}

Une revue des différentes utilisations de la RV met en évidence tout le spectre de ses applications possibles : ludique, professionnelle pédagogique. Adaptée aux digital natives que sont les apprenant·e's d'aujourd'hui, la RV possède l'avantage pédagogique d'expérimenter de voir et de manipuler des espaces et des objets abstraits. Pourtant, peu de projets à vocation pédagogique ont été développés dans le secteur de la visualisation spatiale et de l'architecture.

En cours de développement, l'application GédeRéV permet aux étudiant·e $s$ de se plonger dans l'espace de projection géométral et ainsi de faire l'expérience « sensible » du lien qu'entretiennent les objets représentés et leur représentation. Ceci leur permet d'améliorer leur vision dans l'espace, condition nécessaire à la maîtrise du dessin d'architecture et à une conception rigoureuse des ouvrages.

Remerciements spéciaux aux fonds de développement pédagogique (FDP2018) de l'UCLouvain. 


\section{Bibliographie}

Cache, B., (2016). Toujours l'informe : Géométrie d'Albrecht Dürer, Lausanne, PPUR.

Difede, J. and Hoffman, H.G. (2002). Virtual reality exposure therapy for World Trade Center post-traumatic stress disorder: a case report. CyberPsychology \& Behavior, 5(6), 529-535.

Dorta, T., \& Kinayoglu, G., \& Hoffmann, M. (2014). Hyve-3D: A New Embodied Interface for Immersive Collaborative $3 D$ Sketching.

Fuchs, P., (2016) Les casques de réalité virtuelle et de jeux vidéo, Paris, Presses des MINES, collection Mathématiques et informatique.

Fuchs, P., (2018) Théorie de la réalité virtuelle. Les véritables usages, Paris : Presses des MINES, collection Mathématiques et informatique.

García, R., Quirós, J., Santos, R., et al., (2007), Interactive multimedia animation with macromedia flash in descriptive geometry teaching. Computers \& Education, vol. $49, \mathrm{n}^{\circ}$ 3, pp. 615-639

Gittler, G., Glück, J., (1998), Differential Transfer of Learning: Effects of Instruction in Descriptive Geometry on Spatial Test Performance, dans Journal of Geometry and Graphics, vol. 2, n 1, pp. 71-84.

Greenwald, W., Kulik, A., Kunert, A., et al., (2017) Technology and Applications for Collaborative Learning in Virtual Reality, Philadelphia, International Society of the Learning Sciences, coll. «CSCL 2017 Proceedings ».

Gwee, H., (2013) « Effects of Virtual-Reality Elements on Spatial Visualization Skills of Secondary Three Students in Singapore, dans Cai, Y., édit., 3D Immersive and Interactive Learning, Singapore, Springer, pp. 47-57.

Jaques, D. et Calame, J., (2013), Géométrie spatiale : Le vade-mecum, Lausanne, PPUR. Kim Y, Kim H, Kim YO, (2017), Virtual Reality and Augmented Reality in Plastic Surgery: A Review. Arch Plast Surg. 44(3):179-187.

Martín-Erro, A., \& Espinosa, M.M., \& Domínguez, M. (2014). Industrial applications of virtual reality environments and augmented reality. Dyna, Bilbao, 89. 382-386.

Merchant, Z., Goetz, T., Cifuentes, L., et al., (2014) Effectiveness of virtual reality-based instruction on students' learning outcomes in K-12 and higher education: A metaanalysis, dans Computers \& Education, n ${ }^{\circ} 70$, pp. 29-40.

McVeigh-Schultz, J., \& Márquez Segura, E., \& Merrill, N., \& Isbister, K. (2018). What's It Mean to "Be Social" in VR?. Mapping the Social VR Design Ecology. 289-294.

Monge, G., (1811) Géométrie Descriptive, Paris, J. Klostermann Fils.

O'Connor, M., Deeks, H., Dawn, E., Metatla, O., (2018), Sampling molecular conformations and dynamics in a multiuser virtual reality framework, Science Advances 2018, Vol. 4, no. 6.

Pavel, P., Et Ribeiro Pola, M., (1998), Situations d'apprentissage multimondes dans un système hypermédia sur la géométrie descriptive, dans Rouet, J. et De La Passadière, B., édit., Quatrième colloque "Hypermédias et Apprentissages", Poitiers, EPI-INRP.

Rheingold, H., (1993) La Réalité virtuelle, Paris, Dunod, 1993.

Spiegel, B., Fuller, G., Lopez, M., Dupuy, T., Noah, B., Howard, A., et al. (2019) Virtual reality for management of pain in hospitalized patients: A randomized comparative effectiveness trial.

Zeile, P., Zelling, I., (2019), Virtual Design and BIM in Architectural and Urban Design Potential Benefit for Urban Emotions Initiative, Real Corp 2019 Proceedings, p.841850. 